\title{
A NOTE ON SOME NEW FINITE DIVISION RING PLANES
}

\author{
BY
}

R. SANDLER

In $[1 ; 3]$, certain finite nonassociative division algebras are defined and their autotopism groups determined. In this note, we generalize the definition of [1] and show that some of the new algebras are not isotopic with any of the old algebras. Thus, some new projective planes coordinatized by division rings will appear, although the new planes are very similar in collineation group structure with the planes coordinatized by the old algebras.

Let $K$ be the finite field of $p^{m}$ elements, $p$ prime, $K=G F\left(p^{m}\right)$. Then $K$ is cyclic over $F=G F(p)$, and the automorphism $T$ defined by

$$
x T=x^{p}
$$

generates the automorphism group of $K$ over $F$. Furthermore, $T^{m}=I$. If $x, y$ are in $K$, we shall write

$$
x y=x R(y)=y L(x) .
$$

We shall define a class of algebras as follows: Let $k$ be an integer $1 \leqq k<m$ and let $S=T^{k}$. The algebra $\Omega_{\delta, k}$ for $\delta \neq 0$ in $K$ is the vector space direct sum

$$
K+\lambda K
$$

of two copies of $K$. Multiplication is defined by

$$
(x+\lambda y)(u+\lambda v)=[x u+\delta(y S) v]+\lambda[y u+(x S) v]
$$

for $x, y, u, v$ in $K$. If $L_{x+\lambda y}^{\delta, k}$ is taken to represent left multiplication in $\Omega_{\delta, k}$, then by (3), we have

$$
L_{x+\lambda y}^{\delta, k}=\left(\begin{array}{ll}
R(x) & R(y) \\
R[\delta(y S)] & R[x S]
\end{array}\right) .
$$

Now, $\Omega_{\delta, k}$ will be a division algebra if and only if $L_{z}^{\delta, k}$ is nonsingular for every $z \neq 0$ in $\Omega_{\delta, k}$; i.e., if and only if $x(x S)-\delta y(y S) \neq 0$ unless $x=y=0$. Thus $\Omega_{\delta, c}$ is a division algebra if and only if $\delta \neq c(c S)$ for any $c \in K$. But let $\omega$ be a primitive element of $K$, and let $\omega^{\nu}=\delta$. Then $\delta \neq c(c S)$ if and only if $\omega^{\nu} \neq \omega^{\left(1+p^{k}\right) \mu}$ for any $\mu$ where $\omega^{\mu}=c$. This condition is equivalent to $\mu\left(1+p^{k}\right) \not \equiv \nu\left(\bmod p^{m}-1\right)$ for any $\mu$, which happens if and only if $\left(1+p^{k}, p^{m}-1\right)$ does not divide $\nu$.

In [1], the above algebras were studied in the case where $k$ divides $m$, and the autotopism groups were determined. It will now be shown that the

Received by the editors October 2, 1961. 
above defined class of algebras constitutes a true generalization and that new finite projective planes arise as a result.

The algebras $\Omega_{\delta, k_{1}}$ and $\Omega_{\epsilon, k_{2}}$ are said to be isotopic if there exist three nonsingular, linear transformations of $\Omega_{2}$ onto $\Omega_{1}, Q, P, U$, such that

$$
P L_{x Q}^{\delta, k_{1}}=L_{x}^{\epsilon, k_{2}} U \quad \text { for all } x \text { in } \Omega_{2} .
$$

The significance of the isotopy question is due to the theorem [2, Theorem 6] that two division algebras coordinatize isomorphic projective planes if and only if the algebras are isotopic.

To study the isotopy question, we need to know something about the middle nuclei of the algebras under consideration. The middle nucleus $N_{m}$ of $\Omega$ is the set of all $a \in \Omega$ such that

$$
(x \circ a) \circ y=x \circ(a \circ y)
$$

for all $x, y \in \Omega$. It is known (see [3]) that the middle nucleus of any $\Omega_{\boldsymbol{z}, k}$ is isomorphic with $K$; i.e., is the set of all $x+\lambda \cdot 0, x \in K$. Finally, the following result will be needed (see [4] for a proof): Let $\Omega_{1}, \Omega_{2}$ be two isotopic division algebras. For any $c$ in the middle nucleus of $\Omega_{2}$, there is an element $a$ in the middle nucleus of $\Omega_{1}$ such that

$$
P L_{a}^{1}=L_{c}^{2} P,
$$

where $L^{1}$ and $L^{2}$ represent left multiplication in $\Omega_{1}$ and $\Omega_{2}$, respectively.

Let $S_{1}=T^{k_{1}}, S_{2}=T^{k_{2}}$. Assume now that $\Omega_{\delta, k_{1}}$ and $\Omega_{\epsilon, k_{2}}$ are isotopic. Then there exists a triple $Q, P, U$, satisfying (5). The mapping $P$ can be written in the form

$$
P=\left(\begin{array}{ll}
H_{11} & H_{12} \\
H_{21} & H_{22}
\end{array}\right)
$$

where every $H_{i j}$ is a linear transformation of $K$ over $G F(p)$; i.e.,

$$
H_{i j}=R\left(a_{i j}^{0}\right)+T R\left(a_{i j}^{1}\right)+T^{2} R\left(a_{i j}^{2}\right)+\cdots+T^{m-1} R\left(a_{i j}^{m-1}\right),
$$

$a_{i j}^{\boldsymbol{k}} \in K$. We can rewrite (7), then, as

$$
\left(\begin{array}{cc}
H_{11} & H_{12} \\
H_{21} & H_{22}
\end{array}\right)\left(\begin{array}{cc}
a & 0 \\
0 & \left(a S_{1}\right)
\end{array}\right)=\left(\begin{array}{cc}
c & 0 \\
0 & \left(c S_{2}\right)
\end{array}\right)\left(\begin{array}{ll}
H_{11} & H_{12} \\
H_{21} & H_{22}
\end{array}\right) .
$$

But (10) is equivalent to the relations:

$$
\begin{aligned}
H_{11} R(a) & =R(c) H_{11}, \\
H_{12} R\left(a S_{1}\right) & =R(c) H_{12}, \\
H_{21} R(a) & =R\left(c S_{2}\right) H_{21}, \\
H_{22} R\left(a S_{1}\right) & =R\left(c S_{2}\right) H_{22} .
\end{aligned}
$$


But this implies [1, Lemma 2] that each $H_{i j}=U_{i j} R\left(h_{i j}\right)$ where $U_{i j}$ is an automorphism of $K$, and $h_{i j}$ an element of $K$. Setting $H_{11}=V R\left(h_{11}\right)$, we can write

$$
\begin{aligned}
& H_{12}=V S_{1} R\left(h_{12}\right), \\
& H_{21}=V S_{2}^{-1} R\left(h_{21}\right), \\
& H_{22}=V S_{1} S_{2}^{-1} R\left(h_{22}\right) .
\end{aligned}
$$

Now in (5), let $x=1$, and see that $U=P L_{1 Q}^{1}$. Thus (5) is equivalent to

$$
P L_{x Q}^{1}=L_{x}^{2} P L_{1 Q}^{1}
$$

Writing $1 Q=u_{0}+\lambda \iota_{1}$ and using (11), we can write (12) as

$$
\begin{aligned}
& \left(\begin{array}{cc}
V R\left(h_{11}\right) & V S_{1} R\left(h_{12}\right) \\
V S_{2}^{-1} R\left(h_{21}\right) & V S_{1} S_{2}^{-1} R\left(h_{22}\right)
\end{array}\right)\left(\begin{array}{cc}
a_{0} & a_{1} \\
\delta\left(a_{1} S_{1}\right) & \left(a_{0} S_{1}\right)
\end{array}\right) \\
& =\left(\begin{array}{cc}
x_{0} & x_{1} \\
\epsilon\left(x_{1} S_{2}\right) & \left(x_{0} S_{2}\right)
\end{array}\right)\left(\begin{array}{cc}
V R\left(h_{11}\right) & V S_{1} R\left(h_{12}\right) \\
V S_{2}^{-1} R\left(h_{21}\right) & V S_{1} S_{2}^{-1} R\left(h_{22}\right)
\end{array}\right)\left(\begin{array}{cc}
u_{0} & u_{1} \\
\delta\left(u_{1} S_{1}\right) & \left(u_{0} S_{1}\right)
\end{array}\right),
\end{aligned}
$$

where $\left(x_{0}+\lambda_{0} x_{1}\right) Q=a_{0}+\lambda a_{1}$ for $x_{0}+\lambda_{0} x_{1} \in \Omega_{\epsilon, k_{2}}$. Multiplying, we obtain:

$$
\begin{array}{r}
V R\left(h_{11} a_{0}\right)+V S_{1} R\left(h_{12} \delta\left(a_{1} s_{1}\right)\right)=V R\left[\left(x_{0} V\right) h_{11} u_{0}\right]+V S_{1} R\left[\left(x_{0} V S_{1}\right) h_{12} \delta\left(u_{1} S_{1}\right)\right] \\
+V S_{2}^{-1} R\left[\left(x_{1} V S_{2}^{-1}\right) h_{21} u_{0}\right]+V S_{1} S_{2}^{-1} R\left[\left(x_{1} V S_{1} S_{2}^{-1}\right) h_{22} \delta\left(u_{1} S_{1}\right)\right] \\
V R\left(h_{11} a_{1}\right)+V S_{1} R\left(h_{12}\left(a_{0} S_{1}\right)\right)=V R\left[\left(x_{0} V\right) h_{11} u_{1}\right]+V S_{1} R\left[\left(x_{0} V S_{1}\right) h_{12}\left(u_{0} S_{1}\right)\right] \\
+V S_{2}^{-1}\left[\left(x_{1} V S_{2}^{-1}\right) h_{21} u_{1}\right]+V S_{1} S_{2}^{-1}\left[\left(x_{1} V S_{1} S_{2}^{-1}\right) h_{22}\left(u_{0} S_{1}\right)\right] .
\end{array}
$$

Now, the case $S_{1}=S_{2}$ has essentially been studied in [1], so the assumption is made here that $S_{1} \neq S_{2}$. Also, $S_{1} \neq I \neq S_{2}$. Thus we obtain, by equating distinct automorphisms on both sides of the equality signs,

$$
\left(x_{1} V S_{1} S_{2}^{-1}\right) \delta h_{22}\left(u_{1} S_{1}\right)=0,
$$

$\left(x_{1} V S_{1} S_{2}^{-1}\right) h_{22}\left(u_{0} S_{1}\right)=0$, all $x_{1} \in K$. Since $\delta \neq 0, h_{22}\left(u_{1} S_{1}\right)=h_{22}\left(u_{0} S_{1}\right)=0$. Since everything in sight must be nonsingular, $u_{0}=u_{1}=0$ cannot hold. Hence, $h_{22}=0$. If we further assume that $S_{1} \neq S_{2}^{-1}$, we can write

$$
\left(x_{1} V S_{2}^{-1}\right) h_{21} u_{0}=0=\left(x_{1} V S_{2}^{-1}\right) h_{21} u_{1}
$$

for all $x_{1}$ in $K$. Hence $h_{21} u_{0}=h_{21} u_{1}=0$. But this implies that either $u_{0}=u_{1}=0$ or $h_{21}=0$. In the former case, $1 Q=0$; in the latter, $P$ is singular since $h_{22}=0$, a contradiction. Hence, we may conclude that if $\Omega_{\delta, k_{1}}$ and $\Omega_{\epsilon, k_{2}}$ are isotopic and $S_{1} \neq S_{2}$, then $S_{1}=S_{2}^{-1}$.

On the other hand, if $S_{1}=S_{2}^{-1}$, the equation

$$
\left(\begin{array}{ll}
0 & 1 \\
1 & 0
\end{array}\right)\left(\begin{array}{cc}
a_{0} & a_{1} \\
\delta\left(a_{1} S_{1}\right) & \left(a_{0} S_{1}\right)
\end{array}\right)=\left(\begin{array}{cc}
x_{0} & x_{1} \\
\epsilon\left(x_{1} S_{2}\right) & \left(x_{0} S_{2}\right)
\end{array}\right)\left(\begin{array}{ll}
0 & 1 \\
1 & 0
\end{array}\right)
$$


has a solution if $\delta=\left(\epsilon S_{1}\right)^{-1}$; then $a_{0}=\left(x_{0} S_{2}\right)$ and $a_{1}=\epsilon\left(x_{1} S_{2}\right)$. Thus

$$
P=\left(\begin{array}{ll}
0 & 1 \\
1 & 0
\end{array}\right), \quad Q=\left(\begin{array}{cc}
S_{2} & 0 \\
0 & S_{2} R(\epsilon)
\end{array}\right), \quad U=P .
$$

We have thus proved the following result:

THEOREM. The algebra $\Omega_{\epsilon, k_{2}}$ is isotopic with some $\Omega_{\delta, k_{1}}$ if and only if $S_{1}=S_{2}$ or $S_{2}^{-1}$, i.e., if and only if $k_{1}=k_{2}$, or $k_{1}=m-k_{2}$.

An immediate corollary of the theorem is that if the algebras of [1] are defined with $S=T^{k}, k$ arbitrary, not necessarily dividing $m$, new projective planes arise which are not isomorphic with the planes obtained when $k \mid m$.

The question of the structure of the collineation groups of the new planes arises and it should be pointed out that the methods of [1] are wholly applicable in the case of these more general algebras. The following lemma will be of assistance in studying the collineation groups (see [5] for a proof).

Lemma. If $(r, n)=1, r<n, q$ a prime power, then

$$
\left(1+q^{r}, q^{n}-1\right)=\left(1+q, q^{n}-1\right) .
$$

\section{REFERENCES}

1. A. A. Albert, On the collineation groups of certain non-desarguesian planes, Portugal. Math. 18 (1959), 207-224.

2. - Finite division algebras and finite planes, Proc. Sympos. Appl. Math. Vol. 10, pp. 53-70, Amer. Math. Soc., Providence, R. I., 1960.

3. D. R. Hughes and E. Kleinfeld, Seminuclear extensions of Galois fields, Amer. J. Math. 82 (1960), 389-392.

4. R. Sandler, Autotopism groups of some finite non-associative algebras, to appear in Amer. J. Math.

5. - The collineation groups of some finite projective planes, (to appear).

InSTITUTE For DefEnSE ANALyses,

Princeton, New Jersey 